\title{
Efeitos do treinamento resistido sobre a força muscular e a autopercepção de saúde em idosas
}

\section{Effets of rejstancetrainingonmesdestrengthand thesdf-percedion of hedthin ddely wonen}

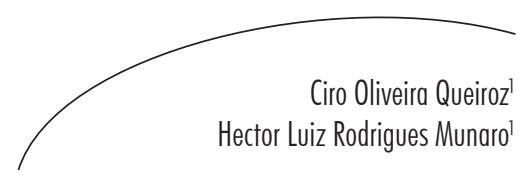

Resumo

Introdução: $\mathrm{O}$ treinamento de força tem sido eficiente em idosas, mas sua relação com a autopercepção de saúde ainda não está clara na literatura. Objetivo: O presente estudo teve como objetivo analisar os efeitos de um programa de treinamento com pesos sobre a força muscular e a autopercepção de saúde em idosas na cidade de Jequié-BA.

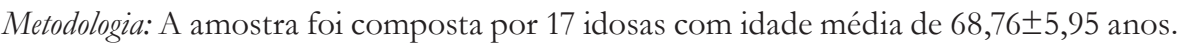
A força muscular foi avaliada pelo teste de estimativa de uma repetição máxima (1RM); o treinamento foi realizado em duas sessões semanais, compostas de duas séries de 10 RM para cada um dos exercícios, com intensidade variando de 50\% a 70\% de $1 \mathrm{RM}$ e duração de oito semanas. Os dados foram analisados por meio da estatística descritiva, do teste t de Student pareado e do teste Qui-quadrado não-paramétrico, com nível de significância de $\mathrm{p}<0,05$. Resultados: A força muscular aumentou significativamente $(\mathrm{p}<0,05)$, houve também aumento na proporção de idosas que relataram autopercepção de saúde positiva, mas não houve diferença estatisticamente significativa após o treinamento. Conclusão: Pode-se concluir que o método de treinamento de força aplicado promoveu respostas significativas no aumento da força muscular e mudanças positivas na autopercepção de saúde referida pelas idosas.

\section{Abstract}

Introduction: The strength training has been efficient in elderly women, however its relationship with the self-perception of health is not yet clear in the literature. Objective: This study aimed to examine the effects of a training program with weights on muscle strength and the self-perception of health in elderly women in the city of Jequié-BA. Methodology: The sample was composed of 17 elderly women with a mean age of $68.76 \pm 5.95$ years. The muscle strength was evaluated by the estimating test of maximum repetition $(1 \mathrm{MR})$. The training was performed in two weekly sessions, composed of two sets of 10 MR for each exercise, with intensity ranging from $50 \%$ to $70 \%$ of $1 \mathrm{MR}$ and duration
Palavras-chave:

Treinamento de resistência. Envelhecimento. Saúde do idoso. Condições de saúde. Jequié-BA.

Key words: Resistance Training. Aging. Health of the Elderly. Health Status. Jequié-BA.

1 Curso de Educação Física. Núcleo de Estudos em Atividade Física e Saúde. Universidade Estadual do Sudoeste da Bahia. Jequié, BA, Brasil. 
of eight weeks. Data were analyzed by descriptive statistics, paired Student t-test and Chi-square non-parametric test with a significance level of $\mathrm{p}<0.05$. Results: The muscle strength increased significantly $(\mathrm{p}<0.05)$, there was also an increase in the proportion of elderly women people who reported positive self-perception of health, however there was no statistically significant difference after the training. Conclusion: It was concluded that the strength training method promoted significant answers on the increase in muscle strength and positive changes on self-perception of health in elderly women.

\section{INTRODUÇÃO}

O envelhecimento é um processo complexo que envolve muitas variáveis (genética, estilo de vida, doenças crônicas) que interagem entre si e influenciam significativamente o modo como alcançamos determinada idade. A participação em programas de atividade física regular (exercícios aeróbio e de força) fornece um número de respostas favoráveis que contribuem para o envelhecimento saudável.

A utilização dos exercícios com pesos em um programa de atividade física regular bem estruturado para preservar a saúde, melhorar a aptidão física e como recurso para o tratamento de patologias, tem sido documentada. ${ }^{1-3}$ Recente estudo mostrou o efeito de 16 semanas de treinamento de força, três vezes por semana, com intensidade de $60 \%$ a $80 \%$ de uma repetição máxima (1RM) em idosas, tendo sido observados aumentos na massa muscular e força máxima, indicando que o treinamento de força pode ser aplicado na reabilitação ou prevenção da sarcopenia de idosas. ${ }^{4}$

A caracterização das condições de saúde dos idosos requer informações detalhadas sobre diferentes aspectos da vida desses indivíduos. Nos países desenvolvidos, é crescente o número de investigações que abordam as associações entre a saúde dos idosos e os determinantes demográficos e socioeconômicos, as doenças crônicas e a capacidade funcional. Uma forma de conduzir esse tipo de estudo é o levantamento de informações sobre a percepção dos idosos em relação a seu próprio estado de saúde. ${ }^{5}$ Obtidas por meio de uma única questão que pede para o indivíduo classificar seu estado de saúde em excelente/muito bom, bom, regular e ruim, a autopercepção de saúde capta, além da exposição a doenças (diagnosticadas ou não por profissionais de saúde), o impacto que tais doenças geram no bem-estar físico, mental e social dos indivíduos.

Pode-se observar que, na literatura, a autopercepção de saúde não é comparada com programas de treinamento de força para idosos, onde esses programas, na maioria das vezes, têm o intuito de melhorar a saúde da população. Percebe-se que a autopercepção de saúde pode ser uma variável para a avaliação de saúde após programas de treinamento de força para idosos.

Este trabalho teve como objetivo analisar os efeitos de um programa de treinamento com pesos sobre a força muscular e a autopercepção de saúde em idosas na cidade de Jequié, Bahia, Brasil.

\section{METODOLOGIA}

\section{Caracterização da amostra}

Antes da aplicação do treinamento, realizouse uma visita a dois grupos de convivência da terceira idade no bairro Jequiezinho, no município de Jequié-BA, para distribuição de fôlderes, e também foram afixados cartazes informando e convidando as idosas a participarem da pesquisa. A população dos dois grupos totalizava 78 idosas, mas 25 mostraram interesse em realizar o programa de treinamento resistido. Com isso, a amostra foi constituída por 25 idosas, voluntárias, que nunca realizaram o treinamento 
resistido. Oito delas desistiram por motivos particulares. A pesquisa foi iniciada, então, com 17 idosas na faixa etária de 61 a 82 anos, com

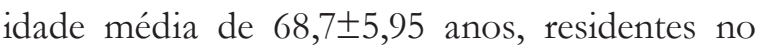
município de Jequié-BA, caracterizando-se como uma amostra intencional e não-probabilística. As idosas apresentaram altura média de 1,52 $\pm 0,05$ $\mathrm{m}$, peso médio de $64,7 \pm 12,45 \mathrm{Kg}$ e índice de massa corporal médio de $27,7 \pm 4,28 \mathrm{Kg} / \mathrm{m}^{2}$.

O estudo foi avaliado e aprovado pelo Comitê de Ética em Pesquisa com Seres Humanos da Universidade Estadual do Sudoeste da Bahia, sob o protocolo número 051/2007.

\section{Procedimentos de coleta de dados}

Para a coleta de dados, todos os voluntários assinaram o termo de consentimento livre e esclarecido e responderam a um questionário sobre a autopercepção de saúde antes e após o treinamento prescrito. Esse instrumento foi previamente testado com base em outro instrumento já validado. ${ }^{7}$ A autopercepção de saúde foi mensurada com base na questão "Como você classifica seu estado de saúde atual?" e tinha com opção de respostas uma escala de quatro pontos (excelente/muito boa, boa, regular e ruim).

O treinamento consistiu na realização de cinco exercícios, três para membros superiores (supino reto, flexão de cotovelo e extensão de cotovelo) e dois para membros inferiores (leg-press e cadeira extensora). Os exercícios foram realizados em máquinas da marca Physicus. O treinamento foi realizado em duas séries de dez repetições com um minuto de intervalo entre as séries, duas vezes por semana, em dias alternados, durante oito semanas. Foi dividido em dois grupos de nove e oito idosas por sessão, para que pudesse haver uma devida atenção, com duração de 30 minutos por sessão. O treinamento foi realizado na sala de musculação da Universidade Estadual do Sudoeste da Bahia, no campus de Jequié-BA.

A intensidade do treinamento foi de $50 \%$ de uma repetição máxima (1RM) na primeira semana, $60 \%$ de 1 RM da segunda até a quarta semana, $50 \%$ de $1 \mathrm{RM}$ na quinta semana e de $70 \%$ de 1 RM da sexta até a oitava semana, resultando assim em um treinamento ondulatório. Foi escolhido este método de treinamento porque a literatura científica na área de envelhecimento ainda carece de resultados de treinamento com alterações flutuantes de volume e intensidade. $\mathrm{Na}$ forma ondulatória, as cargas sofrem uma diminuição periódica antes de um novo pico de carga. Este tipo de treinamento faz com que o músculo suspenda sua atividade e com isso detém a degradação ativa de suas substâncias, os processos de ressíntese se iniciam com tanta intensidade que os recursos energéticos gastos durante certo período depois do trabalho alcançam um nível mais alto do que o inicial. Este estado é conhecido como fase de supercompensação. ${ }^{8}$

Para a avaliação da força muscular máxima, foi empregado o teste da estimativa de uma repetição máxima (1RM). ${ }^{9}$ Segue a equação para essa estimativa:

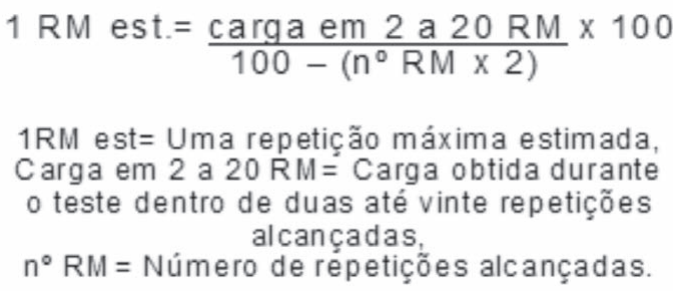

Figura 1 - Fórmula da estimativa de 1 RM.

$\mathrm{Na}$ estimativa de 1RM não foi demonstrada dificuldade na realização dos testes aplicados: quando uma idosa ultrapassava as 20 repetições, o teste era interrompido e se dava um intervalo de cinco minutos para refazê-lo, até alcançar a sua carga máxima.

Antes da execução de cada exercício, foram demonstrados o movimento correto de cada aparelho, a musculatura envolvida e os possíveis sinais de dores músculo-articulares. Antes das sessões dos exercícios, foi realizada uma série dos exercícios para aquecimento neuromuscular sem a utilização de carga. 
Tratamento estatístico

Para a análise estatística dos dados, utilizouse o pacote estatístico SPSS for Windows versão 11.5. Foram adotados para análise e interpretação dos dados um intervalo de confiança de $95 \%$ e nível de significância de 5\% $(\mathrm{p}<0,05)$. Os dados coletados foram analisados por meio da estatística descritiva (média, desvio-padrão e frequência). Para a estatística inferencial, foram utilizados o teste $t$ de Student pareado para as variáveis de força muscular e o teste Qui-quadrado não-paramétrico $\left(\chi^{2}\right)$ para a variável de autopercepção de saúde, visto que não se observou normalidade nos dados por meio do teste Shapiro-Wilk.

A variável relativa à autopercepção de saúde foi dicotomizada e foram considerados indivíduos com saúde positiva aqueles que responderam excelente/muito boa e boa, e os indivíduos com saúde negativa foram aqueles que responderam regular e ruim.

\section{RESULTADOS}

A tabela 1 ilustra os resultados descritivos relacionados à saúde positiva e negativa autoreferidas pelas idosas. Pode-se observar, na tabela, os valores pré e pós-treinamento, o valor do teste estatístico e o nível de significância. Percebe-se que com o treinamento de força houve um aumento de 17,7 pontos percentuais (p.p) na proporção de idosas que relataram a saúde positiva, sem diferença estatisticamente significativa.

Tabela 1 - Autopercepção de saúde pré e pós-treinamento. Jequié, BA, 2008.

\begin{tabular}{lcccccc}
\hline Variável & \multicolumn{2}{c}{ Pré-Treinamento } & \multicolumn{2}{c}{ Pós-Treinamento } & $\chi^{2}$ & $\mathrm{P}$ \\
\multicolumn{1}{c}{ Saúde Hoje } & $\%$ & $\mathrm{n}$ & $\%$ & $\mathrm{n}$ & & \\
\hline Positiva & 58,8 & 10 & 76,5 & 13 & 2,941 & 0,230 \\
Negativa & 41,2 & 7 & 23,5 & 4 & & \\
\hline
\end{tabular}

Na tabela 2, são apresentados os resultados relacionados aos exercícios supino reto, leg press, cadeira extensora, extensão de cotovelo e flexão de cotovelo. Pode-se observar os resultados pré e pós-treinamento, o desvio padrão e o nível de significância.
Percebe-se que todos os exercícios apresentaram um aumento estatisticamente significativo pós-treinamento, sendo a extensão do cotovelo $(69,8 \%)$ e o leg press $(69,5 \%)$ os exercícos que obtiveram o maior aumento nos níveis de força.

Tabela 2 - Resultado médio da comparação dos testes de 1RM (kg). Jequié, BA, 2008.

\begin{tabular}{lcc}
\hline \multicolumn{1}{c}{ Exercícios } & Pré-Treinamento & Pós-Treinamento \\
\hline Supino Reto & $16,72 \pm 4,23$ & $22,87 \pm 4,93 *$ \\
Leg Press & $160,05 \pm 56,80$ & $271,25 \pm 78,08 *$ \\
Cadeira Extensora & $19,92 \pm 5,75$ & $25,38 \pm 5,34 *$ \\
Extensão de Cotovelo & $6,55 \pm 2,27$ & $11,12 \pm 3,07 *$ \\
Flexão de Cotovelo & $14,06 \pm 3,40$ & $17,78 \pm 4,26 *$ \\
\hline
\end{tabular}

* Diferenças significativas entre os momentos pré e pós-treinamento $(\mathrm{p}<0,05)$. 


\section{DISCUSSÃO}

Com o intuito de verificar a reprodutibilidade e aplicabilidade das medidas, foi realizado estudo piloto com o questionário referente à autopercepção de saúde, que demonstrou bons índices de reprodutibilidade..$^{10}$ Esse instrumento apresenta as seguintes limitações: a entrevista pode apresentar imprecisões nas respostas, mesmo que o instrumento tenha sido testado em estudo-piloto e as pessoas nem sempre têm avaliações precisas de seus comportamentos.

Os achados sobre a saúde positiva na tabela 1 assemelham-se com outros estudos realizados por Virtuoso Júnior, ${ }^{11}$ que encontrou 64,7\% de saúde positiva, e também por Ramos. ${ }^{12}$ Salienta-se que esses estudos não ocorreram com treinamento contra resistência, mas com o mesmo público-alvo.

Em outro estudo realizado por Lima-Costa et al., ${ }^{13}$ foram encontrados dados como saúde positiva de $24,7 \%$. Alves \& Rodrigues ${ }^{5}$ também encontraram dados inferiores, se comparados ao presente estudo, sendo que esses estudos também não foram realizados com idosos em fase de treinamento de força. Em estudo recente com a população idosa, também foram encontrados dados relacionados à saúde positiva inferior $(33,8 \%)$ do que o encontrado. ${ }^{14}$ Os estudos aqui discutidos, relacionados à autopercepção de saúde, têm o carater epidemiológico, já que existe carência de estudos que analisam intervenções do treinamento resistido com a avaliação da autopercepção do estado de saúde em idosas.

O presente estudo não demonstrou diferenças estatisticamente significativas; no entanto, apresentou um pequeno aumento nos pontos percentuais com relação ao treinamento de força com efeitos positivos na autopercepção de saúde referida, podendo-se verificar assim uma tendência.

Williams et al., ${ }^{15}$ em recente publicação, mostraram resultados positivos $(p<0,05)$ no aumento da força muscular para extensão e flexão de joelhos e também para a redução de fatores de riscos metabólicos e cardiovasculares em uma intervenção de base comunitária durante 16 semanas na Austrália. Estudos também mostram que o treinamento de força diminui os níveis de lipoproteinas ${ }^{16}$ e melhora a qualidade de vida de idosos com HIV ${ }^{17}$ além de aumentar a força muscular siginificativamente. Percebe-se que o treinamento de força também pode diminuir fatores de risco para a saúde. No presente estudo, não se avaliaram os benefícios do treinamento de força sobre os fatores de risco, mas sim uma simples questão da autopercepção de saúde que pode servir para a avaliação da saúde de idosos.

No que diz respeito ao treinamento de força, a frequência de três dias por semana é bastante utilizada nos protocolos de treinamento de força para idosos. ${ }^{18}$ No entanto, no presente estudo utilizou-se a frequência de dois dias semanais, com um intervalo de 72 horas. Stadler et al. ${ }^{19}$ compararam, em seu estudo, a frequência de dois e três dias de treino e não encontraram diferenças significativas.

Esses dados explicam os resultados encontrados com um protocolo de treinamento de dois dias semanais, segundo o qual ocorreu aumento na força máxima entre $26,4 \%$ e $69,8 \%$ (supino reto $=36,9 \%$, leg press $=69,5 \%$, cadeira extensora $=27,4 \%$, extensão de cotovelo $=69,8 \%$ e flexão de cotovelo $=26,4 \%$ ). Carvalho et al. ${ }^{20}$ também encontraram resultados significativos e semelhantes com frequência de duas vezes semanais, mas esse programa de treinamento teve duração de seis meses.

Um estudo realizado por Rhodes et al. ${ }^{21}$ encontrou dados significativos de força máxima, observando até $36 \%$ de aumento da força muscular em um treinamento na forma de circuito. Esses mesmos estudos foram realizados três vezes por semana, durante 12 meses. No presente estudo, os resultados foram superiores, com frequência semanal de dois dias, durante oito semanas, e encontrando valores de cerca de $69,8 \%$ no aumento da força máxima. Percebe-se que o treinamento realizado de forma ondulatória pode maximizar o efeito da força muscular nas idosas.

O estudo de Vale et al., ${ }^{22}$ com frequência de duas vezes semanais, demonstrou similaridades dos dados aqui encontrados. Demonstrou-se 
também similaridade com dados encontrados por Silva et al., ${ }^{23}$ em que todos os exercícios tiveram aumentos significativos $(p<0,05)$. Nos resultados do estudo realizado por Trancoso \& Farinatti, ${ }^{24}$ também foram observados resultados inferiores aos do presente estudo para o leg press $(58 \%)$ e superior para o supino reto (61\%), sendo que no presente estudo se encontrou cerca de $69,5 \%$ para o leg press e 36,9\% para o supino reto.

Vincent \& Braith $^{25}$ encontraram ganhos de força com $50 \%$ de $1 \mathrm{RM}(52 \%)$ e com $80 \%$ de 1RM (79\%). Karabulut et al. ${ }^{26}$ também encontraram melhores resultados $(\mathrm{p}<0,05)$ trabalhando com $80 \%$ de $1 \mathrm{RM}$ para membros inferiores. Percebe-se que, quando trabalhados com $80 \%$, os resultados encontrados foram maiores do que os do presente estudo, e quando com menor intensidade, $50 \%$ demonstraram similaridade em alguns exercícios. Entretanto, em recente publicação realizada na Espanha por Serra-Rexach et al. ${ }^{27}$ com um protocolo de treinamento de $30 \%$ a $70 \%$ de $1 \mathrm{RM}$ com idosos acima de 90 anos, encontrou-se um aumento na força estatisticamente significativo para membros inferiores.

Em recente estudo realizado na Áustria com intensidade moderada $(50 \%$ a $60 \%$ de 1RM), realizando dez exercícios para membros superiores e inferiores por sessão, foram encontrados resultados estatisticamente

\section{REFERÊNCIAS}

1. Chodzko-Zajko WJ, Proctor DN, Fiatorone Singh MA, Minson CT, Nigg CR, Salem GJ, et al. Exercise and Physical Activity for Older Adults. Med Sci Sports Exerc. 2009;41(7):1510-30.

2. Raso V. Exercício com pesos para pessoas idosas baseado em evidências: Parte I - Segurança, avaliação pré-participação e fatores de risco para doenças cardiovasculares. Rev Bras Ciên e Mov. 2006;14(3):87-96

3. Guido M, Lima RM, Benford R, Leite TKM, Pereira RW, Oliveira RJ. Efeitos de 24 semanas de treinamento resistido sobre índices de aptidão aeróbia de mulheres idosas. Rev Bras Med Esporte. 2010;16(4):259-263. significativos após o treinamento para todos os grupos musculares envolvidos. ${ }^{28}$ Esses dados corroboram o aqui encontrado, em que se verificou aumento estatisticamente significativo para todos os cinco exercícios realizados.

Com relação à intensidade, Raso et al. ${ }^{29}$ realizaram estudo com intensidade de 55\% e encontraram ganhos de força significativos de até $48 \%$. Já no presente estudo, em que foi realizado um treinamento ondulatório variando de $50 \%$ a $70 \%$, foram encontrados dados superiores, chegando-se até cerca de 69,8\%, o que demonstra, assim, que o método de treinamento ondulatório pode ser efetivo no aumento da força muscular em idosas.

\section{CONCLUSÃO}

Pode-se concluir que o método de treinamento de força aplicado neste estudo proporcionou mudanças importantes nos níveis de força muscular máxima em mulheres acima de 60 anos. Também houve mudanças na proporção de idosas no que se refere à autopercepção de saúde positiva; no entanto, não foi observada diferença estatística.

Recomenda-se realizar estudos adicionais com amostra representativa, com grupo controle e maior tempo de treinamento, a fim de se verificar possíveis efeitos positivos.

4. Orsatti FL, Nahas EAP, Maesta N, Nahas-Neto J, Burini RC. Plasma hormones, muscle mass and strength in resistance-trained postmenopausal women. Maturitas. 2008;59(4):394-404.

5. Alves LC, Rodrigues RN. Determinantes da autopercepção de saúde entre idosos do Município de São Paulo, Brasil. Rev Panam Salud Pública. 2005;17(5/6):333-341.

6. Brasil. Ministério da Saúde. Secretaria de Vigilância em Saúde. Secretaria de Gestão Estratégica e Participativa. Vigitel Brasil 2009: vigilância de fatores de risco e proteção para doenças crônicas por inquérito telefônico. Brasília-DF: Editora MS, 2010. 
7. Nahas MV, Fonseca AS. Estilo de Vida e Lazer dos Trabalhadores da Indústria Catarinense (1999-2004). Florianópolis: SESI, 2004.

8. Gomes AC. Treinamento desportivo: estruturação e periodização. 2 ed. Porto Alegre: Artmed, 2009.

9. Adams GM. Exercise Physiology Laboratory Manual. Boston, 1998.

10. Queiroz CO, Munaro HLR. Reprodutibilidade de um instrumento para avaliar os aspectos sócio-demográficos e autopercepção de saúde em indivíduos idosos. Rev Saúde.com. 2008;4(suppl.1):13.

11. Virtuoso Júnior JS. Atividade Física Habitual e Autonomia Funcional de Idosos em Florianópolis, SC. [Dissertação de Mestrado - Programa de PósGraduação em Educação Física]. Florianópolis (SC): Universidade Federal de Santa Catarina, 2004.

12. Ramos LR. A saúde do Idoso no Brasil: uma visão clínico-epidemiológica. [Tese de Doutorado Setor de Geriatria e Gerontologia]. São Paulo (SP): Universidade Federal de São Paulo, 1997.

13. Lima-Costa MF, Firmo JOA, Uchôa E. A estrutura da auto-avaliação da saúde entre idosos: projeto Bambuí. Rev Saúde Pública. 2004;38(6):827-834.

14. Virtuoso Júnior JS, Guerra RO. Incapacidade funcional em mulheres idosas de baixa renda. Ciênc Saúde Coletiva. 2011;16(5):2541-48.

15. Wiliams AD, et al. Cardiovascular and metabolic effects of community based resistance training in an older population. J Sci Med sport. 2011;14(4):331-337.

16. Wooten JS, Philips MD, Mitchell JB, Patrizi R, Pleasant RN, Hein RM, et al. Resistance exercise and lipoproteins in postmenopausal women. Int J Sports Med. 2011;32(1):7-13.

17. Souza PML, Jacob Filho W, Santarém JM, Zomignan AA, Burattini MN. . Effect of progressive resistance exercise on strength evolution of elderly patients living with HIV compared to healthy controls. Clinics. 2011;66(2):261-266.

18. Anton MM, Spriduoso WW, Tanaka H. Age-related declines in anaerobic muscular performance: weightlifting and powerlifting. Med Sci Sports Exerc. 2004;36(1):143 - 47.

19. Stadler LV, Stubbs NB, Vukovich MDA. Comparison of a 2-day and 3-day per week resistance training program on strength gains in older adults(abstract) Med Sci Sports Exerc. 1996;29(5):254.

20. Carvalho J, Oliveira J, Magalhães J, Ascensão A, Mota J, Soares JMC. Força muscular em idosos II - Efeito de um programa complementar de treino na força muscular de idosos de ambos os sexos. Rev Port Cien Desp. 2004;4(1):58-65.

21. Rhodes EC, Martin AD, Taunton JE, Donnelly M, Warren J, Elliot J. Effects of one year of resistance training on the relation between muscular strength and bone density in elderly women. Br J Sports Med. 2000;34(1):18-22.

22. Vale RGS, Barreto ACG, Novaes JS, Dantas EHM. Efeitos do treinamento resistido na força máxima, na flexibilidade e na autonomia funcional de mulheres idosas. Rev Bras Cineantropom Desempenho Hum. 2006;8(4):52-8.

23. Silva CM, et al. Efeitos do treinamento com pesos, prescrito por zona de repetições máximas, na força muscular e composição corporal em idosas. Rev Bras Cineantropom Desempenho Hum. 2006;8(4):39-45.

24. Trancoso ESF, Farinatti PTV. Efeitos de 12 semanas de treinamento com pesos sobre a força muscular em mulheres com mais de 60 anos de idade. Rev Paul Educ Física. 2002;16(2):220-29.

25. Vincent KR, Braith RW. Resistance exercise and bone turnover in elderly men and women. Med Sci Sports Exerc. 2002;34(1):17-23.

26. Karabulut M, Abe T, Sato Y, Bemben MG. The effects of low-intensity resistance training with vascular restriction on leg muscle strength in older men. Eur J Appl Physiol. 2010;108(1):147-155.

27. Serra-Rexach JA, Bustamante - Ara N, Hierro Villarán M, González Gil P, Sanz Ibáñez MP, Blanco Sanz N, et al. Short-term, light- to moderateintensity exercise training improves leg muscle strength in the oldest old: a randomized controlled trial. J Am Geriatr Soc. 2011;59(4):594-602.

28. Berent R, von Duvillard SP, Crouse SF, Sinzinger $\mathrm{H}$, Green JS, Schmid P. Resistance training dose response in combined endurance-resistance training in patients with cardiovascular disease: a randomized trial. Arch Phys Med Rehabil. 2011;92(10):1527-1533.

29. Raso V, Benard G, Duarte AJS, Natale VM. Effect of resistance training on immunological parameters of healthy elderly women. Med Sci Sports Exerc. 2007;39(12):2152- 59. 